\title{
Isolated neurosarcoidosis presenting with recurrent hydrocephalus
}

\author{
Frederick L. Hitti ${ }^{1}$, Benjamin C. Kennedy ${ }^{1}$, Yazmin Odia ${ }^{2}$, Claire S. Riley ${ }^{3}$, Sameer A. Sheth ${ }^{1}$ \\ ${ }^{1}$ Department of Neurological Surgery, Neurological Institute, Columbia University Medical Center, New York, NY 10032, USA. \\ ${ }^{2}$ Department of Neuro-Oncology, Neurologic Institute of New York, Columbia University College of Physicians and Surgeons, \\ New York, NY 10032, USA. \\ ${ }^{3}$ Department of Neurology, Neurologic Institute of New York, Columbia University College of Physicians and Surgeons, New York, \\ NY 10032, USA.
}

\section{A B S T R A C T}

Sarcoidosis is an inflammatory process that is characterized by the formation of noncaseating granulomas. This protean disease may afflict nearly any organ system, including the central nervous system. Here, we present a case of isolated neurosarcoidosis that initially presented with hydrocephalus requiring ventriculoperitoneal shunt placement. The patient's hydrocephalus recurred multiple times and required two additional shunt placements over the 3-year course of her illness. Due to the lack of systemic involvement, sarcoidosis was only diagnosed after a tissue biopsy of a Cauda equine lesion. This case highlights the importance of tissue diagnosis and the diagnostic workup for sarcoidosis in cases of cryptogenic hydrocephalus.

Key words: Biopsy, hydrocephalus, inflammatory, neurosarcoidosis

\section{INTRODUCTION}

Sarcoidosis is an inflammatory disease that may present in a multitude of forms due to its ability to involve virtually any organ in the body. Involvement of the central nervous system (CNS) occurs in 5-25\% of sarcoidosis patients. ${ }^{[1-3]}$ The most common symptoms associated with neurosarcoidosis are cranial nerve palsies, paresthesia, headache, weakness, ataxia, aseptic meningitis, peripheral neuropathy, and cognitive impairment [Table 1]. Hydrocephalus is a much rarer complication of neurosarcoidosis that was not found in one series of 91 cases and only reported in a few case reports. ${ }^{[4,7-10]}$ Neurosarcoidosis patients commonly have evidence of the involvement of the thoracopulmonary system. Isolated neurosarcoidosis (sarcoidosis limited solely to the nervous system) is relatively rare. The prevalence

Corresponding Author: Dr. Frederick L. Hitti,

Department of Neurological Surgery, Neurological Institute,

Columbia University Medical Center, 4th Floor, 710,

West 168th Street, New York, NY 10032, USA.

E-mail: fhitti@gmail.com

\begin{tabular}{|l|l|}
\hline \multicolumn{2}{|c|}{ Access this article online } \\
\hline Quick Response Code: & \\
\hline & Website: \\
\hline & www.nnjournal.net \\
\cline { 2 - 2 } & DOI: \\
\hline
\end{tabular}

of isolated neurosarcoidosis among neurosarcoid patients varies from series to series with older studies reporting $1-3 \%$ and more recent studies reporting $11-17 \% .^{[4,11]}$ Here, we report a case of a patient with isolated neurosarcoidosis that presented with hydrocephalus ultimately requiring placement of three ventriculoperitoneal shunts and eventually diagnosed by biopsy of Cauda equina lesions.

\section{CASE REPORT}

A 38-year-old African-American woman presented to an outside hospital with a 2-year history of headaches, a 5-month history of difficulty walking, dizziness refractory to meclizine, and a 3-day history of blurry vision. A neurological exam demonstrated nystagmus on upgaze, left gaze, and right gaze. She also had an ataxic gait and papilledema. Head computed tomography (CT), and magnetic resonance imaging (MRI) revealed communicating hydrocephalus. Analysis of cerebrospinal fluid (CSF) obtained by lumbar puncture (LP) demonstrated cell count, protein,

This is an open access article distributed under the terms of the Creative Commons Attribution-NonCommercial-ShareAlike 3.0 License, which allows others to remix, tweak, and build upon the work non-commercially, as long as the author is credited and the new creations are licensed under the identical terms.

For reprints contact: nn_editor001@nnjournal.net

Cite this article as: Hitti FL, Kennedy BC, Odia Y, Riley CS, Sheth SA. Isolated neurosarcoidosis presenting with recurrent hydrocephalus. Neuroimmunol Neuroinflammation 2015;2:287-90.

Received: 15-04-2015; Accepted: 06-08-2015 
and glucose levels within normal limits. A presumptive diagnosis of idiopathic hydrocephalus was made. A right frontal external ventricular drain was placed followed by right frontal ventriculoperitoneal shunt placement. A postoperative head CT revealed a collapsed right lateral ventricle and unchanged left lateral, third, and fourth ventricles. Due to symptomatic improvement, the unresolved hydrocephalus was followed without further intervention.

Five months later, the patient presented to the outside hospital with a headache, nausea, vomiting, ataxia, dizziness, and blurred vision. Other than an opening pressure of $25 \mathrm{~cm}$ of $\mathrm{H}_{2} \mathrm{O}$ and glucose of $97 \mathrm{mg} / \mathrm{dL}$, CSF analysis was within normal limits. Angiotensin-converting enzyme (ACE) levels were not tested. Head CT revealed left unilateral

\begin{tabular}{lcc}
\hline \multicolumn{3}{l}{ Table I: Signs and symptoms of neurosarcoidosis } \\
\hline Sign or symptom & Percent affected (\%) & References \\
\hline Cranial neuropathy & $52-73$ & {$[3,4-6]$} \\
Aseptic meningitis & $7-24$ & {$[3,4-6]$} \\
Peripheral neuropathy & $6-24$ & {$[3-6]$} \\
Cognitive impairment & $2-27$ & {$[4,5]$} \\
Seizures & $2-20$ & {$[4-6]$} \\
Myopathy & $9-12$ & {$[3,6]$} \\
CNS space occupying lesion & $2-11$ & {$[4-6]$} \\
HPA axis dysfunction & $2-11$ & {$[4-6]$} \\
Hydrocephalus & $4-9$ & {$[3,6]$} \\
Paresthesia & 43 & {$[4]$} \\
Headache & 37 & {$[4]$} \\
Weakness & 33 & {$[4]$} \\
Ataxia & 24 & {$[6]$} \\
Myelopathy & 21 & {$[4]$} \\
Encephalopathy & 11 & {$[6]$} \\
Hemiparesis & 7 & {$[4]$} \\
Guillain-Barré syndrome & 5 & {$[5]$} \\
Radiculopathy & 3 & {$[4]$} \\
\hline
\end{tabular}

CNS: central nervous system; HPA: hypothalamic pituitary adrenal obstructive hydrocephalus secondary to a trapped ventricle with a left-to-right midline shift, so a left frontal ventriculoperitoneal shunt was placed. Although postoperative MRI revealed decreased hydrocephalus, the third and fourth ventricles remained prominent. No postcontrast enhancement was seen [Figure 1a and b].

Other than one shunt revision, she remained relatively stable for 3 years after which she presented to the outside hospital with unsteadiness, double vision, and left leg weakness. Head CT revealed collapsed lateral and third ventricles and a persistent dilatation of the fourth ventricle, which prompted placement of a ventriculoperitoneal shunt in the fourth ventricle. She was discharged, but her condition continued to deteriorate, possibly due to over-shunting or disease progression and she was readmitted after 2 weeks. At this point, she transferred her care to our institution.

Upon arrival, her neurological exam demonstrated direction-changing nystagmus, asymmetric proximal lower extremity weakness (iliopsoas right $3 / 5$, left $2 / 5$ ), and ataxia on finger-nose-finger and heel-shin testing. There were no sensory deficits, and the patient did not complain of fecal or urinary incontinence. Head CT demonstrated bilateral frontal and right suboccipital approach ventricular catheters and a decompressed ventricular system. Hypo-density, suggestive of edema, in the cerebellum prompted treatment with Decadron. MRI revealed sulcal enhancement bilaterally along the frontal lobes and internal auditory canals that suggested leptomeningeal disease [Figure 1c and d]. MRI of the spine revealed diffuse nodular leptomeningeal enhancement throughout the spinal
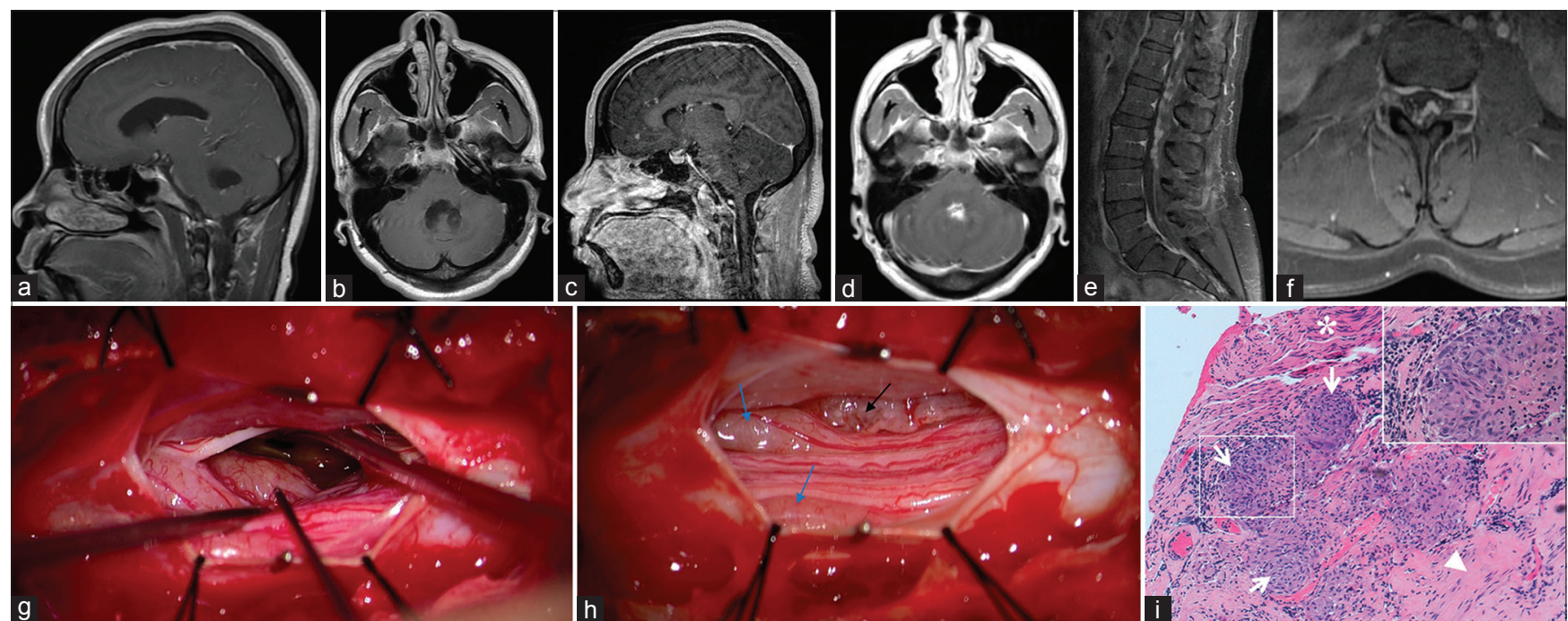

Figure 1: (a and b) Outside institution T1 postcontrast magnetic resonance imaging (MRI) (5 months after initial presentation); (c-f) T1 postcontrast MRI obtained upon admission to our institution (3.5 years after initial presentation) that demonstrates leptomeningeal enhancement throughout the central nervous system; ( $g$ and $\mathrm{h}$ ) Intraoperative photographs of the exposed nerve roots at the L3 level of the Cauda equina; (g) Micrograph before the biopsy. The yellow-gray, nodular, hyper-vascular lesion intimately related to the leptomeninges that was biopsied is marked by a black stimulation probe; (h) Micrograph demonstrating the lesion after biopsy (black arrow) and two lesions (blue arrows) that produced motor potentials upon stimulation; (i) Photomicrograph depicting confluent nonnecrotizing granulomas (arrows) involving fibrocollagenous tissue of leptomeninges (arrowhead), and nerve fibers (asterisk); $\mathrm{H}$ and E, $\times 100$. Inset shows one highlighted granuloma at $\times 400$ 
cord and Cauda equina [Figure 1e and f]. Opening pressure was normal following LP and CSF analysis revealed lymphocytic pleocytosis (88 WBCs/uL, 94\% lymphocytes), markedly elevated protein ( $868 \mathrm{mg} / \mathrm{dL})$, and an ACE level of $15 \mathrm{U} / \mathrm{L}$ (normal, 0-2.5 U/L). Cytology demonstrated lymphocytosis without malignant cells, and flow cytometry was negative for lymphoma. The workup for infection was negative. Chest X-ray and CT of the abdomen and pelvis were negative for mass lesions or signs of systemic sarcoidosis. She experienced only transient improvement on steroids, so they were tapered off.

Discussion at a multidisciplinary case conference yielded a broad differential diagnosis that included inflammatory processes (neurosarcoidosis or other connective tissue diseases), primary or secondary CNS neoplasm (ependymoma and lymphoma), or a chronic infectious leptomeningitis. Biopsy from the L3 Cauda equina region was recommended as the lowest risk and highest yield target. A region of a root that did not produce a motor potential when stimulated was biopsied [Figure 1g and h]. Hematoxylin and eosin stained sections demonstrated numerous noncaseating granulomas [Figure 1i]. No organisms were identified with acid-fast or Grocott's methenamine silver stains. These biopsy findings support a diagnosis of neurosarcoidosis.

\section{DISCUSSION}

Here, we describe a case of isolated neurosarcoidosis presenting with hydrocephalus that over three years appeared to require three separate shunt catheters, likely due to progressive occlusion of the foramina of Monro and Sylvian aqueduct or possibly due to elevated CSF protein levels. Hydrocephalus is a rare symptom of neurosarcoidosis with few case reports..$^{[7-10]}$ This is, to our knowledge, the first report of isolated neurosarcoidosis presenting with hydrocephalus. Furthermore, noteworthy is the development of a trapped lateral ventricle and necessity of multiple shunt placements. While there has been one report of neurosarcoidosis requiring multiple shunt placements, the patient described had systemic sarcoidosis making diagnosis more straightforward. ${ }^{[8]}$ The protracted course of our patient's undiagnosed illness was likely due to the absence of systemic disease.

Upon initial presentation, the diagnostic workup, including CSF analysis, was unrevealing, and she was diagnosed with idiopathic hydrocephalus at the outside institution. The leptomeningeal enhancement, later found on MRI, generated a broad differential diagnosis that included infectious, inflammatory, and neoplastic diseases. Due to the protracted course of her illness and the presence of multiple lesions throughout her brain and spinal cord, ependymoma, and neurosarcoidosis were placed highest on the differential diagnosis. Several years into her disease course, her CSF profile became remarkable for the nonspecific findings of lymphocytosis, elevated protein and elevated ACE. . $5,12,13]$ While her CSF analysis was normal early in her disease course, this has been previously reported in other cases of neurosarcoidosis. ${ }^{[6,7]}$

Because the appropriate clinical and radiographic findings are nonspecific, sarcoidosis diagnosis generally requires biopsy. ${ }^{[1-3]}$ For isolated neurosarcoidosis, diagnosis is made even more challenging by the absence of thoracopulmonary involvement, a hallmark present in $90 \%$ of patients. ${ }^{[1,2]}$

Biopsy for sarcoidosis is also critical because patients may not show substantial improvement with an empiric trial of steroids, as was true for this patient. Many have advocated the use of immunosuppressants such as azathioprine, methotrexate, infliximab, or mycophenolate mofetil alone, or in addition to steroids in patients who do not respond to corticosteroid monotreatment. ${ }^{[1,2,14-16]}$ The risks of long-term immunosuppressive therapy warranted a definitive diagnosis of isolated neurosarcoidosis, given its rarity. Since neurosarcoidosis was indeed the diagnosis, the patient was initiated on $80 \mathrm{mg}$ prednisone daily and $500 \mathrm{mg}$ mycophenolate mofetil twice a day.

\section{CONCLUSION}

This report highlights the importance of considering neurosarcoidosis in the differential diagnosis in patients with unexplained recalcitrant hydrocephalus.

Financial support and sponsorship

Nil.

Conflicts of interest

There are no conflicts of interest.

\section{REFERENCES}

1. Hoitsma E, Faber CG, Drent M, Sharma OP. Neurosarcoidosis: a clinical dilemma. Lancet Neurol 2004;3:397-407.

2. Iannuzzi MC, Rybicki BA, Teirstein AS. Sarcoidosis. N Engl J Med 2007;357:2153-65

3. Stern BJ, Krumholz A, Johns C, Scott P, Nissim J. Sarcoidosis and its neurological manifestation. Arch Neurol 1985;42:909-17.

4. Nozaki K, Scott TF, Sohn M, Judson MA. Isolated neurosarcoidosis: case series in 2 sarcoidosis centers. Neurologist 2012;18:373-7.

5. Sharma OP. Neurosarcoidosis: a personal perspective based on the study of 37 patients. Chest 1997;112:220-8.

6. Gascón-Bayarri J, Mañá J, Martínez-Yélamos S, Murillo O, Reñé R, Rubio F. Neurosarcoidosis: report of 30 cases and a literature survey. Eur J Intern Med 2011;22:e125-32. 
7. Akhondi H, Barochia S, Holmström B, Williams MJ. Hydrocephalus as a presenting manifestation of neurosarcoidosis. South Med $J$ 2003;96:403-6.

8. Kim SH, Lee SW, Sung SK, Son DW. Treatment of hydrocephalus associated with neurosarcoidosis by multiple shunt placement. J Korean Neurosurg Soc 2012;52:270-2.

9. Tabuchi S, Uno T. Hydrocephalus with panventricular enlargement as the primary manifestation of neurosarcoidosis: a case report. $J$ Med Case Rep 2013;7:240.

10. van Rooijen JM, Mijnhout GS, Aalders TT, de Bondt RB. Hydrocephalus, a rare manifestation of sarcoidosis. Clin Pract 2011;1:e66.

11. Chapelon C, Ziza JM, Piette JC, Levy Y, Raguin G, Wechsler B, Bitker MO, Bletry O, Laplane D, Bousser MG, Godeau P. Neurosarcoidosis: signs, course and treatment in 35 confirmed cases. Medicine (Baltimore) 1990;69:261-76.
12. Khoury J, Wellik KE, Demaerschalk BM, Wingerchuk DM. Cerebrospinal fluid angiotensin-converting enzyme for diagnosis of central nervous system sarcoidosis. Neurologist 2009;15:108-11.

13. Qian X, Goumnerova LC, De Girolami U, Cibas ES. Cerebrospinal fluid cytology in patients with ependymoma: a bi-institutional retrospective study. Cancer 2008;114:307-14.

14. Androdias G, Maillet D, Marignier R, Pinède L, Confavreux C, Broussolle $\mathrm{C}$, Vukusic $\mathrm{S}$, Sève P. Mycophenolate mofetil may be effective in CNS sarcoidosis but not in sarcoid myopathy. Neurology 2011;76:1168-72.

15. Moravan M, Segal BM. Treatment of CNS sarcoidosis with infliximab and mycophenolate mofetil. Neurology 2009;72:337-40.

16. Scott TF, Yandora K, Valeri A, Chieffe C, Schramke C. Aggressive therapy for neurosarcoidosis: long-term follow-up of 48 treated patients. Arch Neurol 2007;64:691-6. 